\title{
Téoros
}

Revue de recherche en tourisme

\section{Essai d'identification des problématiques de gestion liées à une spécificité sectorielle}

\section{Le cas des produits et des services touristiques}

\section{Michel Balfet}

Volume 17, numéro 3, automne 1998

Pratiques contemporaines de gestion dans le domaine du tourisme

URI : https://id.erudit.org/iderudit/1072163ar

DOI : https://doi.org/10.7202/1072163ar

Aller au sommaire du numéro

Éditeur(s)

Université du Québec à Montréal

ISSN

0712-8657 (imprimé)

1923-2705 (numérique)

Découvrir la revue

Citer cet article

Balfet, M. (1998). Essai d'identification des problématiques de gestion liées à une spécificité sectorielle : le cas des produits et des services touristiques.

Téoros, 17(3), 18-25. https://doi.org/10.7202/1072163ar d'utilisation que vous pouvez consulter en ligne. 


\section{A N A L Y S E}

\section{ESSAI D'IDENTIFICATION DES PROBLÉMATIQUES DE GESTION LIÉES À UNE SPÉCIFICITÉ SECTORIELLE}

LE CAS DES PRODUITS ET DES SERVICES TOURISTIQUES

\section{Michel Balfet}

Parcourir le champ de recherche que le "phénomène tourisme sengendre offre des possibilités d'entrées aussi riches que variées. On peut percevoir le tourisme comme un domaine qui offre des approches interdisciplinaires à la fois qualitatives et quantitatives qui intéressent autant les spécialistes des sciences humaines et sociales que ceux des sciences réputées \& dures . .

Quel que soit le contenu sémantique que chacun donne au mot tourisme, il apparaît comme un fait de société, conséquence de comportements individuels et collectifs. Un économiste, quelque peu sociologue et anthropologue, soulignerait qu'on se trouve le plus souvent confronté à un besoin et à une demande qui trouvent leur origine dans les attitudes et les motivations des individus, mais également dans l'expression des comportements collectifs des membres de nos sociétés. Par ailleurs, on peut percevoir le tourisme comme une spécificité sectorielle, une portion de l'offre de l'appareil de production de biens et de services marchands ou non marchands'. C'est, dans ce cas, une industrie au sens * de processus complet d'élaboration et de mise en marché de chaînes de services $x^{2}$. Cette complexité s'accroît avec l'évolution des exigences de la demande. Ainsi peut-on rappeler deux grandes orientations contemporaines de l'offre touristique induites par la demande : une recherche de gammes de produits-services de plus en plus complètes et intégrées, présentées sous forme de * packages $\%$, ainsi qu'un effort de fiabilité et de continuum de qualité entre des entreprises qui ne relèvent pas toujours de métiers et d'espaces identiques. À une qualité sans faille des prestations fournies par les points de commercialisation des zones émettrices de clientèle doit répondre une fiabilité de leurs prolongements nationaux ou internationaux chargés du « réceptif * de ces mêmes clients.

Le chercheur en sciences de gestion s' intéresse aux approches sociologiques ou anthropologiques relevant du couple marchéproduit autant que par celles qui relèvent de l'étude des systèmes de production liés à l'activité touristique. Il l'est d'autant plus quand la recherche porte sur la rencontre des deux, à savoir l'étude des organisations permettant de rapprocher offre et demande. Toutefois, il rencontrera deux types de difficultés relevant de deux préoccupations complémentaires et interactives : déterminer ce qui, dans l'étude envisagée, relève véritablement de son champ d'analyse et de sa compétence et identifier, parmi les caractéristiques du phénomène touristique étudié, celles qui relèvent d'une véritable spécificité de ce champ.

Afin d'identifier les apports possibles des sciences de gestion à la connaissance de la spécificité tourisme, nous orienterons notre reflexion dans deux directions :

- Une revue succincte et préalable de l'objet des sciences de gestion et des champs qui peuvent y être associés, ceci dans le schéma des organisations opérant dans l'univers du tourisme ;
- Un repérage des spécificités sectorielles propres à l'activité tourisme et une approche des questionnements et des problématiques qui peuvent conduire à une connaissance scientifique des particularités de gestion propres aux organisations dédiées à l'élaboration et à la mise en marché de produits et services touristiques.

\section{OBJET DES SCIENCES DE GESTION ET SPÉCIFICITÉ "TOURISME *}

Désirant souligner par une incidente l'objet des sciences de gestion, nous nous garderons d'aborder un débat épistémologique aujourd'hui très nourri, le plus souvent vif et appuye ${ }^{3}$. Toutefois, un souci de clarté impose de rappeler cet objet par le biais de quelques définitions qui ont en commun la mise en évidence de l'intérêt des sciences de gestion pour l'organisation.

Dès 1981, le professeur Lassegue fut un des premiers à définir les sciences de gestion comme \& l'application des sciences à la conduite des organisations ${ }^{4}$; le professeur Marchesnay les considère actuellement comme $\alpha$ le fait d'engager des moyens, au sein d'un ensemble organisé, aux fins de réaliser des buts que se proposent ceux qui les ont acquis pour les mettre en cuvre $^{5}$. Notre pratique des sciences de gestion nous porte à considérer leur objet comme * l'ensemble 'connaissance - action-Evaluation' ayant pour finalité d'améliorer les performances de l'organisation tout en s'efforçant d'identifier et de prévenir les risques $m^{6}$. Les trois définitions proposées, ainsi qu'une revue d'articles spécifiques consacrés à la gestion 
appliquée au monde du tourisme nous amènent à penser que c'est bien dans les particularités de l'ensemble organisationnel dédié au tourisme et dans l'approche de ses performances et de ses risques que le chercheur en sciences de gestion peut apporter un éclairage utile à la connaissance, à l'explication et à la maîtrise des eléments de la spécificité tourisme.

\section{UNE DÉMARCHE SCIENTIFIQUUE EN SCIENCES DE GESTION}

Plusieurs questionnements sont nécessaires pour asseoir une démarche scientifique en sciences de gestion. L'objectif du chercheur est d'améliorer les performances de l'organisation et d'évaluer le risque qu'elle encourt. Ainsi se trouve-t-il confronté à trois ensembles de préoccupations présentant de fortes interrelations.

Le choix méthodologique est le premier ensemble dans lequel s'inscrit le savoirfaire du chercheur. Toute la capacité scientifique de l'appareil choisi sera portée par la pertinence cognitive, a acquisitive $n$ et décisionnelle des outils mis en ouvre, mais également par les possibilités d'adaptation et de récurrence que l'ensemble méthodologique mobilise et autorise.

Les points d'application de la recherche sur l'ensemble organisationnel constituent le deuxième ensemble de préoccupations. Finalite, acteurs, procedures, structures, feront l'objet de démarches a connaissance-action $x$ dans le cadre d'approches monographiques ou systémiques dont nous apprécierons plus avant la pertinence, Il convient toutefois de souligner le rôle central que cet ensemble joue dans la connaissance d'une spécificité.

L'organisation est, dans la plupart des éventualités, mise en place pour une durée significative; son fonctionnement s'inscrit, à priori, dans la continuité. Les volontés de connaissance et d'action du chercheur se moulent donc dans un tel contexte, d'autant que la boucle recherchevalidation exige du temps. Toutefois, la grande instabilité des sociétés et de leur environnement ainsi que les déséquilibres qui les affectent, amenent le plus souvent à inscrire la participation du gestionnaire comme urgente ${ }^{8}$. Les rythmes d'apprentissage, de compréhension et de contrôle s'en trouvent quelque peu altérés, voire fortement perturbés. Dans le contexte de telles réductions temporelles, seul le cadre d'un processus bouclé, quasi cybernétique, peut apporter une vraie réponse aux impératifs d'amélioration de la performance et d'évaluation du risque.

La globalité de la démarche peut être éclairée par le schéma 1. Par cette représentation, d'inspiration générale, transposable dans son principe à toute organisation, nous avons voulu souligner les points essentiels et les noeuds d'une progression finalisé, bouclé, visant à ameliorer les performances et à prévenir les risques qui pourraient en découler. Ce schéma sera également marqué \& tourisme $x$ dans la mesure où les composantes organisationnelles présentées correspondent à une organisation dédiée à cette activité.

\section{LES DOMAINES D'APPLICATION DE LA DÉMARCHE * SCIENCES DE GESTION *}

Notre approche précisera l'architecture des composantes de l'organisation. Elle necessite quelques compléments analytiques afin de situer les points d'application que Ia démarche précédente a mis en évidence.

L'approche la plus synthétique, la plus globale d'une organisation réside essentiellement dans la finalité que lui ont attribué ses promoteurs. Ceci est particulièrement important dans le référentiel tourisme français où coexistent des volontés très différenciées: finalité commerciale et finalité à vocation associative, voire sociale. C"est probablement à ces niveaux qu'apparaissent les aspects les plus tangibles des choix stratégiques et des orientations politiques des organisations concernées. La finalité ressort donc comme la composante majeure de l'organisation : son respect, qui constitue une fin en soi, génère les règles de positionnement des autres composantes.

Les trois autres composantes constituant la base organisationnelle de l'ensemble se rattachent pour l'essentiel aux logiques du système de production, à savoir les acteurs (clients, employés, actionnaires, tiers), les structures (internes et externes) correspondant à des données liées à l'univers stable de l'organisation et, enfin, les procédures que nous classerons en procédures liéces au système d'information, procédures liées au système de décision et procédures de contrôle. Dans un souci de simplification conceptuelle, nous nous proposons de représenter l'ensemble organisationnel, composantes et liens qui l'unissent, à l'aide d'une figure géométrique à trois dimensions : le tétraèdre.

On peut associer la finalité du systène organisationnel au sommet du tétràdre, les sommets à sa base ếtant les composantes * acteurs $\%$, structures * et * procédures $*$. Les six arêtes représentent les liens

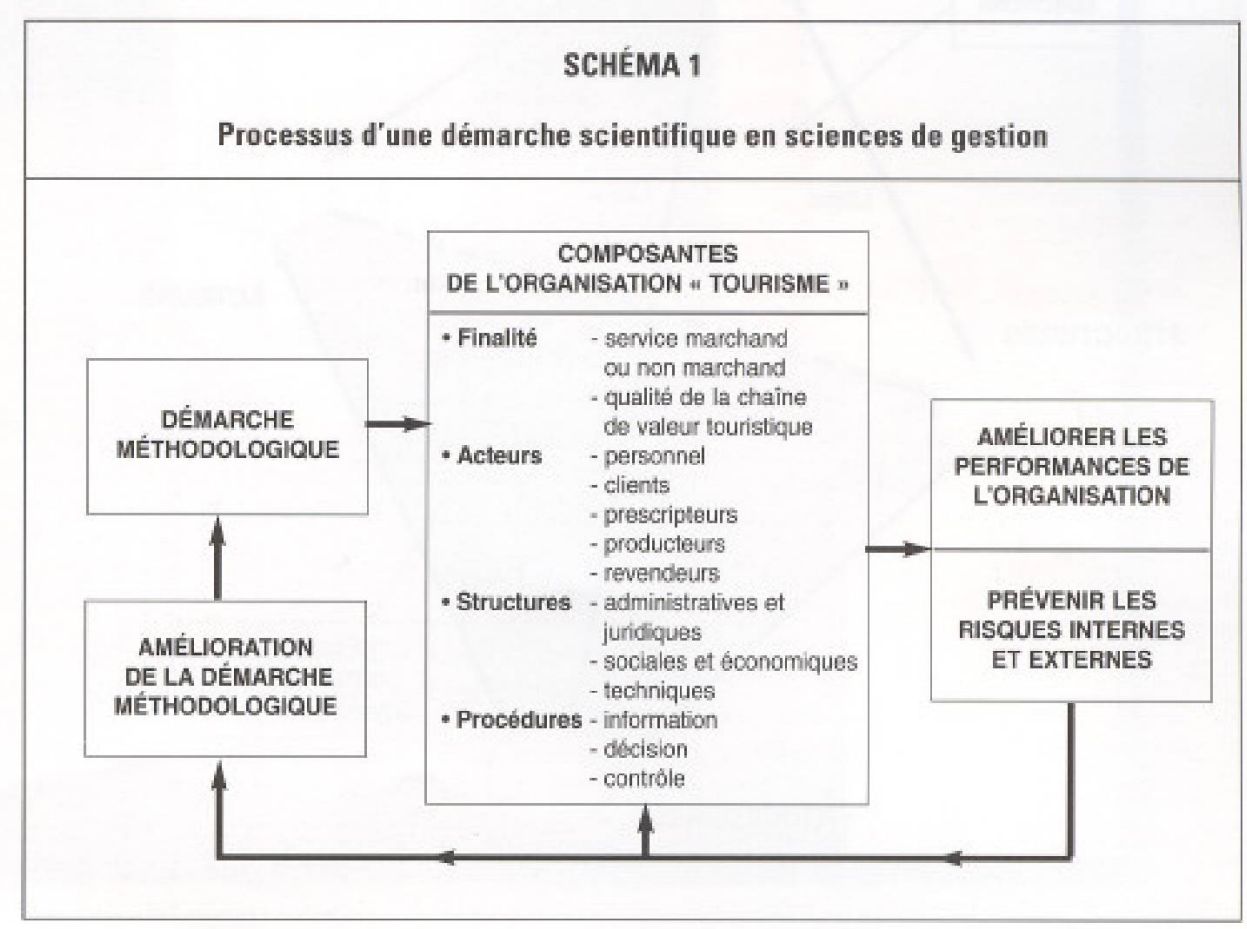


organisationnels générés par la logique du système et peuvent être assimilés à des opérateurs, au sens mathématique du terme, qui lient les composantes organisationnelles deux à deux. Dans leur globalité géométrique, ces liens traduisent les aspects de politique et de stratégie liés à la vie et au développement de l'organisation $^{9}$.

Le schéma qui suit veut traduire la représentation de la réalité organisationnelle composantes-liens.

Transposée au champ de la spécificité tourisme, au tourisme associatif par exemple, la représentation suggérée précédemment peut être la suivante dans l'hypothèse d'un ensemble * village vacances a vocation sociale $*$,

Des considérations de lisibilité nous ont empêché d'ajouter la nature des liens, mais quelques exemples complèteront le schéma.

Exemples de liens :

\section{FINALITÊ $\square$ ACTEURS}

- Application du critère d'admission sociale et familiale à l'instar du critère de solvabilité. Signification particulière de la notion de produit et de client.

- Logement du personnel associatif non rémunéré contre prestations de service.

- Politique de rémunération du personnel local d'animation et de service, décalée par rapport au marché du travail.

\section{FINALITÉ a STRUCTURES}

- Investissement immobilier de capacité moyenne, financé par des institutions et des associations régionales, forte intégration au site.

- Participation de l'association-mère au financement des travaux d'infrastructure logistique (moyens administratifs - matériels - savoir-faire-gestion).

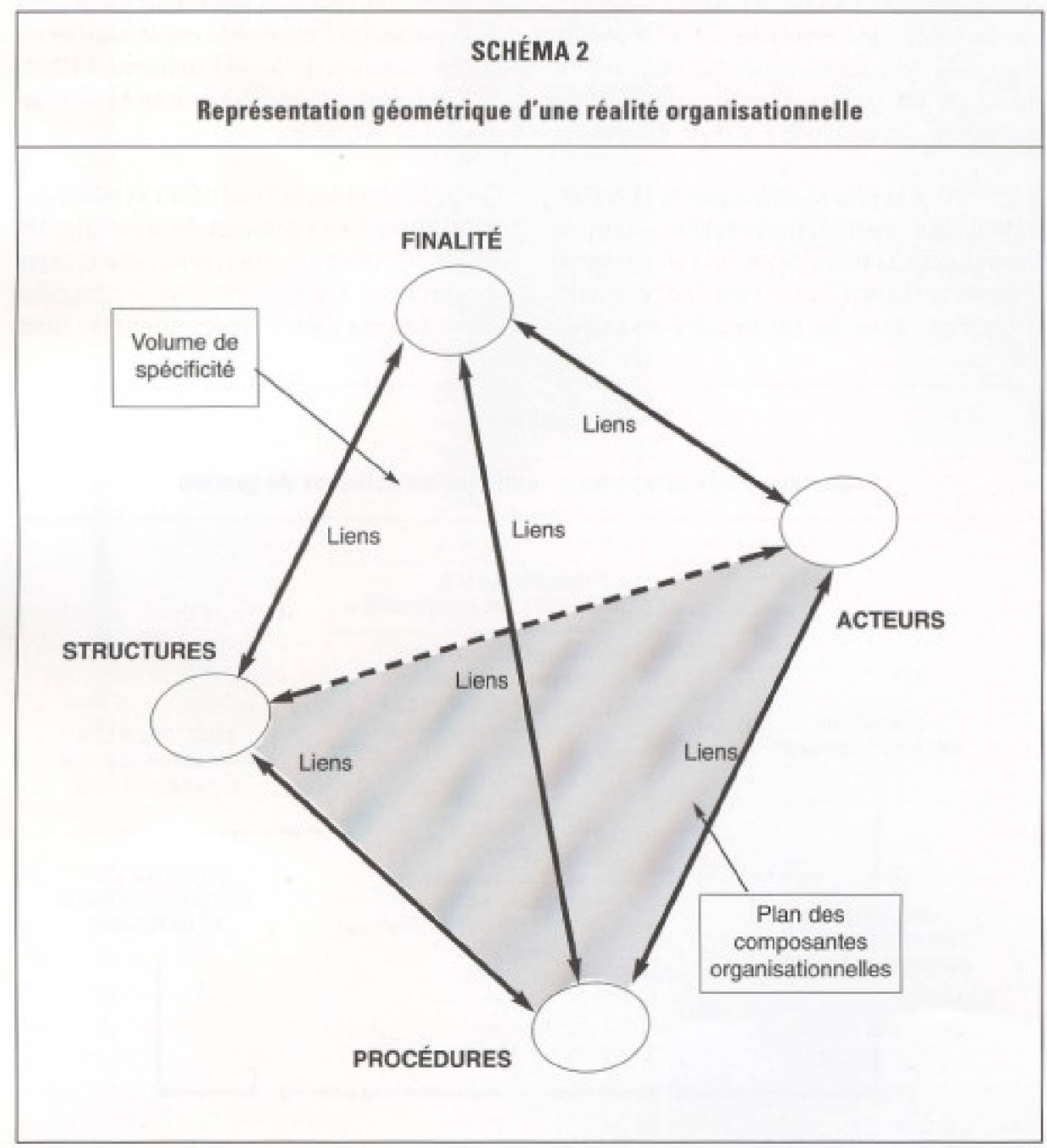

\section{FACTEURS DE SPÉCIFICITÉ ET PROBLÉMATIQUES LIÉES À LA GESTION DES ORGANISATIONS ET DES ENTREPRISES DE TOURISME}

Nous nous sommes efforcés de souligner précédemment les domaines fondamentaux d'application d'une démarche a sciences de gestion $*$ à l'organisation. Que cette dernière soit entreprise, institution publique, privée, ou encore structure libérale, on doit compléter l'approche épistémologique proposée par une confrontation avec les caractères de spécificité issus de ce que l'on pourrait qualifier * d'environnement touristique $*$. Un rapprochement entre les composantes organisationnelles sur lesquelles s'exercera l'action de gestion, a finalite $m, \alpha$ acteurs $m, a$ structures $m$, « procédures $\%$, et les facteurs de spécificité de l'univers du tourisme, peut faciliter l'identification des grandes problématiques liées à la gestion des organisations dédiées au tourisme.

Cette préoccupation fonde notre analyse. 


\section{LES FACTEURS DE SPÉCIFICITÉ QUI CARACTÉRISENT L'UNIVERS DU TOURISME}

On considère comme touriste \& toute personne en déplacement hors de sa résidence habituelle pour une duré d'au moins 24 heures ou une nuit et au plus de 4 mois, pour les motifs suivants : agrément (vacances et séjours de fin de semaine), santé (thermalisme, thalassotherapie), missions et réunions de toutes sortes (congrès, séminaires, pèlerinages, manifestations sportives, etc.), voyages d'affaires et déplacements professionnels, voyages scolaires ${ }^{11}$. Cette définition de la demande touristique s'applique également au tourisme international. Elle porte en elle des balises précises et exhaustives du champ du tourisme qui ouvrent des pistes d'analyses anthropologiques et sociologiques des comportements; en outre, elle facilite une identification des systemes de production liés aux services touristiques.

Le rappel du vocable $a$ services touristi-

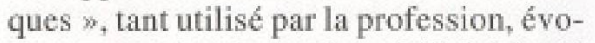
que un premier niveau de spécificité fondé essentiellement sur la nature tertiaire de ce type de prestation. Toutefois, s' arreter à cette seule constatation ne permet pas de constituer une base analytique suffisamment discriminante pour différencier les activités liées au tourisme et pour déterminer des caractères de spécificité véritablement originaux.

Les facteurs de spécificité principalement reconnus par les auteurs spécialisés ont été analysés et classés selon des modalités $\mathrm{d}^{4}$ entrées différentes. \& Les produits touristiques présentent trois caractères principaux : inélasticité, complémentarité et hétérogénéité * ; cette classification économique du professeur F. Vellas ${ }^{12}$ traduit des réalités que nous retrouvons sous forme $\mathrm{d}^{4}$ approche stratégie-politique chez $\mathrm{G}$. Tocquer et M. Zins ${ }^{13}$ : \& le produit touristique est un produit d'une spécificité particulière, et ce, pour plusieurs raisons. Chacun de ces critères confere au produit touristique une nature particulière qui affecte dans une large mesure les stratégies et la commercialisation de celui-ci. Cette spécificité se traduit à partir des critères suivants : la tangibilité et l"intangibilité, la multiplicité des composantes, la multiplicité des intervenants, I'environnement géographique, la multiplicité des types de produits, les caractéristiques d'un service, les caractéristiques d'ordre public et social $\%$. Yves Tinard ${ }^{14}$, pour sa part, lie la spécificité aux caractéristiques des composantes techniques du séjour touristique : transport, hébergement, restauration, animation.

Face à tant d'approches des caractéristiques de l'environnement touristique, nous avons tenté de a donner la parole s aux professionnels par le biais de résultats d'une enquête empirique à vocation exploratoire ${ }^{15}$. Cette enquête, qualitative, réalisée auprès de cadres opérationnels d'entreprises, d'associations ou d'institutions liées au tourisme, a été menée de septembre 1995 à janvier 1996 dans la région Toulouse Midi-Pyrénées ainsi qu'en région parisienne. Construite à partir d'entretiens brefs, peu directifs, elle permet d'éclairer, et le plus souvent de rejoindre, les analyses théoriques présentées précédemment. Son objectif principal est l'identification des principaux facteurs de spécificité liés au produit-service touristique. La méthodologie s'appuie sur deux outils dont le premier, un w questionnaire $s$ ne comportant qu'une seule question qui, après test préalable, a été libellếe ainsi : « Quelles sont, en-dehors des éléments de politique commerciale : prix, enseigne, communication, publicité, la ou les particularités majeures qui influent sur la distribution des produits-services que vous proposez ? \%. A l'issue de la transcription des réponses, un second outil a été utilisé. $\mathrm{A}$ partir des mots clés, à l'aide d'une procédure bayésienne de regroupement idéologique, les idées majeures ont été réduites aे 9,6 , puis 4 thématiques, par ordre de fréquence décroissante d'apparition :

- Thématique 1 - l'aspect le plus souvent mis en évidence par les professionnels est la spécificité a chaîne de produits-services $\%$, de fonctionnement en réseau, de produits agrégés bien qu'hétérogènes. Cette caractéristique de chaîne de fonction, voire de chaîne de valeur, apparaît comme une caractéristique d'évaluation forte $\mathrm{s}$.

- Thématique 2 - un autre aspect nettement suggéré par les responsables interrogés est la confrontation du produitservice touristique au facteur temps. Cet aspect apparaît comme une constante majeure. Caractère saisonnier, cycles hebdomadạires, volatilité et intangibilité, « non-transférabilité * des produits et services touristiques sont les mots cles qui se degagent des transeriptions.
- Thematique 3 - le produit-service touristique, dans sa grande perméabilité aux cadres et aux faits de société, porte une troisidme famille de facteurs de spécificité : les faits politiques, sociaux, économiques, l'évolution des cadres juridiques, sont autant de leviers puissants qui modifient les orientations et les composantes du système de production touristique.

- Thématique 4- la dimension spatiale de l'activité touristique apparaît également comme une caractéristique de spécificité importante. Espace, objet de transaction, espace facilitant la transaction, concentration des lieux touristiques, sont autant de caractéristiques qui émergent des réponses obtenues.

- Thématique résiduelle — un certain nombre de réponses n'ont pu être attribuées à aucune des quatre familles de thematiques retenues ci-dessus.

Nous avons mené l'enquête auprès de 33 professionnels appartenant aux quatre grandes composantes des métiers du tourisme : hôtellerie-restauration (11), agences de voyages et voyagistes (11), activités de transport de voyageurs (5), activités liées à l'animation: culture, santé, sport et aventure (6). L'objectif était de recueillir le point de vue des différentes facettes de l'offre touristique, sans volonté de rechercher un Echantillon strictement représentatif de cette offre (nécessité d'un échantillon nettement plus important, choix de critères de stratification complexes).

Les 33 professionnels ont émis 125 mentions utilisables pour notre analyse, dont 116 ont pu être reclassées selon les 4 thématiques identifiées.

Nous utiliserons, au cours des développements ultérieurs, la réduction lexicale des réponses en quatre thématiques qui deviendront ainsi les quatre spécificités retenues dans notre approche:

- Chaîne de fonction

- Caractère saisonnier - volatilité

- Sensibilité aux faits de société

- Lien organique avec l'espace

Le tableau 1 nous permet de formuler quelques remarques qui portent sur deux aspects induits par les chiffres: I'éventuelle hiérarchisation entre les facteurs ainsi identifiés et les niveaux de dépendance ou 
d'indépendance entre les lignes \& métiers * et les colonnes a facteurs de spécificité \%.

L'identification des facteurs de spécificité était notre objectif et il serait intéressant de hiérarchiser leur importance, mais les chiffres livrés par le tableau ne peuvent répondre à ce type de questionnement car la faiblesse de l'échantillon traité ne peut couvrir toute la complexité qu'une stratification de l'offre aurait nécessité. Toutefois, peut-on avancer sans trop de risques que l'aspect maîtrise de la chaîne de fonction et les aspects maîtrise du caractère saisonnier et de la volatilité du service, constituent les deux paramètres de gestion importants pour les responsables d'organisations qui connaissent ces caractéristiques d'exploitation.

L'architecture du tableau de contingence proposé, croisant a métiers $\$$ avec $\alpha$ spécificités \%, peut inciter à rechercher le niveau de dépendance ou d'indépendance entre lignes et colonnes. Cette recherche se heurtera à quelques éléments qui peuvent être atténués par regroupement de lignes du tableau.

- Neuf cases sur les 20 qui composent le tableau présentent un effectif de mentions inférieur à 5 ; le calcul du khi-deux s'en trouvera affecté.

- Il convient de regrouper les métiers par niveaux de proximités comportementales. Une présentation du tableau en A.F.C., même dans l'hypothèse où le $k h i$-deux n'est pas significatif, situera les proximités et, par là, les lignes à regrouper. Il faudra persévérer dans le sens d'une amélioration du khi-deux. Un regroupement Hôtellerie-Restauration-Animation, d'une part, et Agences de voyages-Voyagistes-Transports, d'autre part, conduit à un khi-deux égal à 4,86 avec $1-\mathrm{p}=81,75 \%$, soit un niveau peu significatif puisqu'il y a $18,25 \%$ de probabilités que la répartition soit due au hasard.

Nous présentons en annexe le calcul du khideux du tableau initial, le graphe d'A.F.C. permettant de situer les proximités ainsi que le calcul du khi-deux du tableau ainsi regroupé. Notons que ces deux aspects peuvent constituer des approches complémentaires à notre propos.

A la suite de ces développements, nous pouvons retenir que l'élaboration d'une

\begin{tabular}{|c|c|c|c|c|c|c|}
\hline \multicolumn{7}{|c|}{$\begin{array}{c}\text { TABLEAU 1 } \\
\text { Effectifs des mentions }\end{array}$} \\
\hline $\begin{array}{l}\text { FACTEURS DE } \\
\text { SPECIFICITE }\end{array}$ & $\begin{array}{l}\text { THËME 1 } \\
\text { CHAIINE } \\
\text { DE } \\
\text { FONCTIONS }\end{array}$ & $\begin{array}{l}\text { THËLE } 2 \\
\text { CARACTERE } \\
\text { SAISONNIER } \\
\text { VOLATILTEE }\end{array}$ & $\begin{array}{l}\text { THËME } 3 \\
\text { SENSIBLILTE } \\
\text { AUX FNTS DE } \\
\text { SOCIEETE }\end{array}$ & $\begin{array}{l}\text { THËEE } 4 \\
\text { LLEN } \\
\text { ORGANIOUE } \\
\text { ANEC LESPACEE }\end{array}$ & AUTHES & TOTAL \\
\hline $\begin{array}{l}\text { HÓTELLERIE } \\
\text { RESTAUAATION } \\
\text { (11) }\end{array}$ & 15 & 11 & 9 & 10 & 3 & 48 \\
\hline $\begin{array}{l}\text { AGENCES DE } \\
\text { WOYAGES } \\
\text { WOYAGISTES } \\
\text { (11) }\end{array}$ & 20 & 11 & 10 & 4 & 5 & 50 \\
\hline $\begin{array}{l}\text { TRANSPOAT } \\
\text { (5) }\end{array}$ & 6 & 4 & 2 & 1 & 1 & 14 \\
\hline $\begin{array}{l}\text { ANIMATION } \\
\text { (6) }\end{array}$ & 4 & 5 & 1 & 3 & 0 & 13 \\
\hline TOTAL & 45 & 31 & 22 & 18 & 9 & 125 \\
\hline
\end{tabular}

Effectif de personnes interrogées : 33

Nombre de mentions relevées : 125

prestation à vocation touristique est fondée sur un ensemble de services, une chaîne de fonctions très sensible aux facteurs temps et espace, ainsi qu'aux caractéristiques juridiques, sociales et politiques de l'espace considéré.

La technologie d'élaboration du service touristique est, dans la majorité des cas, fondée sur une chaîne de fonctions, quasiment une chaîne de valeur au sens de M. Porter ${ }^{16}$. Les métiers des entreprises ou des organisations qui l'animent et la mettent en auvre peuvent être très différents du métier des organisations initiatrices : agents de voyage, associations, et, organismes institutionnels ${ }^{17}$.

Ce premier élément de spécificité, très porteur de particularismes professionnels, correspond en fait au facteur de risque majeur que comporte l'activité d'agent de voyage. L'exemple d'une offre $\alpha$ longue distance s par un agent de voyage, pour un prix forfaitaire comprenant transport, hébergement, restauration, loisirs, visites, services d'un guide, assurance et, éventuellement, moyen de financement, est l'illustration la plus large de cette première particularité ; l'exemple d'un produit hôtelier fondé sur les produits hébergement, boissons, room service, restauration, systèmes de communication, organisation de séminaires et de conférences, peut également être considéré comme une chaîne de valeur, surtout dans l'hypothèse où certains services sont * externalisés \%.

La prestation touristique peut également être perçue dans le cadre d'un repère temps. Au caractère intangible de nombreux services qui la constituent s'ajoute une grande volatilité de l'offre ; la place d'avion qui n'a pas « trouvé preneur $\%$, la chambre d'hôtel inoccupée, la location d'un véhicule non concrétisée, sont autant de ventes perdues qui ne pourront pas être rattrapées. Cette volatilité est par ailleurs fortement accentuée par le caractère saisonnier du phénomène touristique, les caractéristiques des clientèles renforçant ou atténuant cette particularitéts ${ }^{18}$.

Par ailleurs, la prestation touristique est très sensible au poids et à l'évolution des structures mentales, sociales et juridiques. Cette extrême sensibilité aux phénomènes sociaux, économiques et politiques constitue probablement un facteur de spécificité important, souvent sous-estimé par le fait qu'il est diffus ou, au contraire, brutalement ponctuel ; dans cette dernière éventualité, il prend la forme d'accidents sociaux, économiques ou politiques qui peuvent engendrer des risques extrêmes pour le système d'offre de services et, en retour, une quasi disparition de ces derniers ${ }^{19}$.

La variable spatiale (concentration, dispersion) liée à la mise en ceuvre du service 
nous semble être un quatrième facteur de spécificité. L'aspect concentration géographique des points de prestation découle du caractère nécessairement limité des zones dédiées au service à vocation touristique : impératifs technologiques (exemple : station de ski), rareté des sites naturels remarquables, concentrations urbaines liées à l'histoire ancienne ou récente en sont les explications premiêres.

\section{QUESTIONNEMENTS - PROBLÉMATIQUES SPÉCIFIQUES DE GESTION}

Déterminer les questionnements et les pistes pouvant induire une contribution pertinente du spécialiste en sciences de gestion à l'étude de la * spécificité tourisme $x$ nous conduira à mettre en correspondance les composantes organisationnelles évoquées dans notre première partie avec les quatre grands caractères de spécificité identifiés. Ce croisement nous permettra de mieux cerner les nouds d'une problématique et les démarches de connaissance et d'action propres à satisfaire la compréhension de cette spécificité,

\section{VERS UNE APPROCHE OPÉRATIONNELLE}

L'objet de la démarche proposée se veut utilitaire ; ainsi allons-nous privilégier un mode représentatif simple autorisant une lecture directe des observations. Nous travaillerons essentiellement à partir d'un cadre matriciel présentant, en lignes, les composantes organisationnelles : finalité, structures, acteurs et procédures définies plus tôt (dans la section traitant des domaines d'application de la démarche "s sciences de gestion a à l'organisation) et, en colonnes, les quatre facteurs importants de la spécificité des activités liées au tourisme : chaîne de fonction, caractère saisonnier-volatilité, sensibilité aux faits de société, espace. Nous ne prendrons pas en compte le cinquième facteur qualifié de « divers ». Les problématiques, objets de notre identification, figureront dans les cases de la matrice ; la présentation générale du tableau sera donc arrêtée dans la forme proposée au tableau 2.

Le tableau pourra être lu case par case, mais également en regroupant des ensembles de cases correspondant à plusieurs composantes organisationnelles et à plu-

\begin{tabular}{|c|c|c|c|c|}
\hline \multicolumn{5}{|c|}{$\begin{array}{l}\text { TABLEAU } 2 \\
\text { Grille de présentation des problématiques }\end{array}$} \\
\hline $\begin{array}{l}\begin{array}{l}\text { FACTEUAS DE } \\
\text { SPECIFICITE }\end{array} \\
\begin{array}{l}\text { OOMPOSANTES } \\
\text { ORGANISA. } \\
\text { TIONNELLES }\end{array}\end{array}$ & $\begin{array}{l}\text { CHAIINE } \\
\text { DE } \\
\text { FONCTIONS }\end{array}$ & $\begin{array}{l}\text { CARACTEERE } \\
\text { SAISONNIER } \\
\text { WOLATILITE }\end{array}$ & $\begin{array}{l}\text { SENSIBILITE } \\
\text { AUX FAITS } \\
\text { DE SOCIETEE }\end{array}$ & $\begin{array}{l}\text { LIEN OAGANIOUE } \\
\text { AVEC } \\
\text { L'ESPACE }\end{array}$ \\
\hline FINALITE & pb. 11 & pb. 12 & $\mathrm{pb} .13$ & pb. 14 \\
\hline STRUCTURES & pb. 21 & pb. 22 & pb. 23 & ph. 24 \\
\hline ACTEURS & $\mathrm{pb} .31$ & $p b .32$ & pb. 33 & po. 34 \\
\hline PROCÉDURES & pb. 41 & pb. 42 & $\mathrm{pb} .49$ & ph. 44 \\
\hline
\end{tabular}

$\mathrm{pb}=$ problématiques

sieurs facteurs de spécificité. Par agrégation des problématiques présentes dans les cases, pour une organisation donnée, il sera possible de situer les éléments caractéristiques de ses choix politiques et stratégiques. Ainsi serons-nous à mểme de matérialiser la nature des liens du tétraèdre organisationnel présenté plus haut.

\section{LA NATURE DES QUESTIONNEMENTS DE L'ORGANISATION DÉDIÉE AU TOURISME}

Explicitons le mode de construction du tableau. La première ligne constitue le cadrage initial des questionnements retenus. Le rôle primordial dévolu à ces quatre premières cases résulte de la position de la composante \& finalité * dans l"architecture du tétraèdre organisationnel que nouss avons déjà présentée.

Le croisement de la composante finalitế avec chaque grand caractère de spécificité * tourisme $*$ livre quatre grands questionnements clés : performance, fluctuation, conformité, prédétermination.

- Performance : il s'agit bien d'un questionnement majeur eu egard à la technologie d'élaboration du service et à la finalité de l'organisation quelle que soit l'expression de cette performance : profit, croissance, qualité de prestation, etc.;

- Fluctuation : l'immatérialité du produit touristique, son caractère saisonnier, sa volatilité, font que finalité de l'organi- sation et confrontation au facteur temps posent la question importante de la fluctuation des activités, voire des résultats attendus ;

- Conformité : ce noud problématique situe tout le rapport entre finalité et sensibilité sociale du produit touristique ; il est fortement lié aux modes, aux contraintes sociales, aux grands courants d'idées qui touchent les populations des zones émettrices comme celles des zones réceptrices ;

- Prédétermination : le rôle organique de l'espace dans le produit touristique fait que la finalité de ce dernier est liée à une forme de prédétermination. Citons pour exemple des formes de tourisme telles que la croisière, le tourisme urbain, le tourisme montagnard.

Ces questionnements clés doivent aussi être rapprochés des autres composantes organisationnelles que sont les structures, les acteurs, les procédures.

La performance de la chaîne de fonctions et de valeur et, par là, sa contribution à la finalité de l'organisation, sont tributaires du niveau d'insertion de ses structures dans l'architecture et la logique de la chaîne ainsi établie. Elles sont fonction également de l'appropriation de sa réalité opérationnelle par les acteurs, agents de production ou consommateurs, voire tiers intéressés. Enfin, la cohérence des objectifs de la chaîne avec les procédures clés de l'organisation est le dernier élément qui permet de démultiplier l'efficacité de la chainne de fonctions, donc sa potentialité de valeur. 
Lorsqu'on projette le produit touristique dans le temps, une grande fluctuation de la demande semble un élé́ment de spécificité à la fois redouté et apprécié par les professionnels. Les réponses que l'organisation peut apporter à ce phénomène s'inscrivent dans trois sous-ensembles: la flexibilité des structures, l'adaptabilité des acteurs (clients, professionnels ou tiers intéressés) et la recherche de modularité des procédures intéressant l'organisation pour compléter le type de réponse aux fluctuations des marchés touristiques.

La sensibilité et la perméabilité sociale exigent une grande conformité du produitservice touristique avec les structures mentales, sociales, juridiques et politiques locales, nationales et internationales; cette conformité peut être approchée par l'adéquation des structures de l'organisation aux institutions, par une bonne anticipation de l'attitude des acteurs et, probablement, par une recherche de $*$ transférabilité s entre les procédures de l'ensemble organisationnel et son environnement d'accueil.

Le rôle essentiel de la prédétermination géographique dans l'identification du produit-service touristique impose de contrôler trois éléments liés à l'organisation productive : l'existence d'une logistique, complément essentiel de l'action, un comportement adapté des acteurs de l'organisation et des acteurs locaux, ainsi que l'intégration au dispositif local des différentes procédures propres à l'organisation.

\section{* TRANSFÉRABILITÉ * DES PROBLÉMATIQUES GÉNÉRALES IDENTIFIÉES}

Les problématiques générales et les questionnements qui les accompagnent présentent un caractère général peu \& opérationnalisable \& à ce niveau. Deux pistes de développement peuvent être envisagées :

- On peut s"interroger sur les aspects disciplinaires (droit, économie, adminis= tration, gestion) que la grille d'observation proposée peut induire. Au tableau 4 , nous proposons une lecture du cadre 3 orientée dans le sens des sciences de gestion et d'administration appliquées à des organisations productrices de services touristiques.

- On peut également s'interroger sur l'aspect * gestion d'un produit tourisme *

\begin{tabular}{|c|c|c|c|c|}
\hline & $\begin{array}{l}\text { ille des problé } \\
\text { proposa }\end{array}$ & $\begin{array}{l}\text { TABLEAU } \\
\text { atiques posé } \\
\text { des services }\end{array}$ & $\begin{array}{l}\text { une organisatio } \\
\text { ristiques }\end{array}$ & \\
\hline $\begin{array}{l}\text { FACTEURS DE } \\
\text { SPECIFICITE } \\
\begin{array}{l}\text { COMPOSANTES } \\
\text { ORGANISATION- } \\
\text { NELLES }\end{array}\end{array}$ & $\begin{array}{l}\text { CHAINE } \\
\text { DE } \\
\text { FONCTIONS }\end{array}$ & $\begin{array}{l}\text { CARACTERE } \\
\text { SAISONNIER } \\
\text { VOLATILITE }\end{array}$ & $\begin{array}{l}\text { SENSIEIITE } \\
\text { AUX } \\
\text { FAITS DE } \\
\text { SOCIETTÉ }\end{array}$ & $\begin{array}{l}\text { LIEN ORGANIOUE } \\
\text { AVEC } \\
\text { L'ESPACE }\end{array}$ \\
\hline FINALITE & PERFORMANCE & FLUCTUATION & CONFORMITÉ & PRÉDÉTERMINATION \\
\hline STRUCTURES & INSERTION & FLEXIBILITÉ & ADÉQUATION & LOGISTIQUE \\
\hline ACTEURS & APPROPAIATION & ADAPTABILTÉ & ATTITUDE & COMPORTEMENT \\
\hline PROCÉDURES & COHÉRENCE & MODULAFITÉ & TRANSFÉRABILITÉ & INTEGGRATION \\
\hline
\end{tabular}

\begin{tabular}{|l|c|c|c|}
\hline \multicolumn{3}{|c|}{$\begin{array}{c}\text { TABLEAU 4 } \\
\text { Problematique de gestion des services }\end{array}$} \\
\\
s'appliquant au champ de spécificité \& Tourismen
\end{tabular}

que la même grille peut induire. Nous proposons au tableau 5 la transcription du cadre 3 dans l'hypothèse d'un produit de tourisme social et associatif.

Le tableau 4 précise quelques unes des problématiques de gestion pour lesquelles le tourisme correspond à un champ d'application particulier.

\section{CONCLUSION}

L'étude du champ de recherche induit par le « phénomène tourisme * présente pour le praticien des sciences de gestion des possibilités d'entrées aussi variées que riches. L'exploitation d'une telle spécificité est fructueuse par référence à ses caractéristiques majeures : 
- La nature de la prestation touristique dans ses composantes chânées, ses métiers et ses univers:

- La réactivité extrême des systèmes d'élaboration de ces prestations par rapport au temps, à l'espace, aux environ= nements.

Le relevé de telles caractéristiques, toujours en mouvement, conduit à considérer ce champ de spécificité comme fréquemment renouvelé, toujours apte à recevoir les investigations d"un praticien ou d'un chercheur curieux de systémiques complexes.

Michel Balfet est Mâtre de Conferrences habilite a la direction de recherches en Sciences de gestion. Depuis 1993, il dirige le Centre d'Etude du Tourisme et des Industries de l'Accueil (Cetia). Ce centre est organise en département regroupant un I.U.P. (deug, licence, maîtrise), un DESS et un centre international de formation au tourisme et à l'hotellerie. Une equipe de recherche est associée au Cetia.

Un comite de lecture a lu et accepté ce texte

\section{NOTES}

1 L'activitế de services non marchands est particulièrement importante dans le monde du tourisme. Elle regroupe la production relevant du tourisme social et associatif. Consulter à ce propos l'ouvrage du professeur Jean Froidure (1997).

2 Balfet, C., 1994:55.

3 Lemoigne, J. L., 1993 ; Martinet, 1990.

4 Lassegue, 1981.

5 Marchesnay, 1991.

6 Balfet, M., 1994.

7 Cahiers Espaces, (1991): 23 passim.

8 Riveline, 1991.

9 Treboul, $1998 ; 45$.

10 Evrard, Bras, Roux, 1994 : chap. 1.

11 La Documentation Française, $1990: 5$.

12 Vellas, $1992: 96-99$.

13 Tocquer et Zins, $1987: 153-157$.

14 Tinard, $1994: 5=15$.

15 Balfet, M., 1996: 21-25.

16 Porter, 1993.

17 Lafforgue, $1994: 50 \mathrm{~s}$.

18 Chazaud, $1991: 5-7$; Chaspoul, $1991: 8-12$.

19 Un exemple de régression brutale du marché touristique nous est donné par les suites économiques du tragique attentat de Louxor, en Egypte.

\begin{tabular}{|c|c|c|c|c|}
\hline \multicolumn{5}{|c|}{ TABLEAU 5} \\
\hline $\begin{array}{l}\text { FACTEURS DE } \\
\text { SPECIFICITE } \\
\begin{array}{l}\text { COMPOSANTES } \\
\text { ORGANISATION: } \\
\text { NELLES }\end{array}\end{array}$ & $\begin{array}{l}\text { CHAINE } \\
\text { DE } \\
\text { FONCTIONS }\end{array}$ & $\begin{array}{l}\text { CAARGTERE } \\
\text { SAISOMNIER } \\
\text { WOLATILITE }\end{array}$ & $\begin{array}{l}\text { SENSIBILITE } \\
\text { AUX FAITS } \\
\text { DE } \\
\text { SOCIETE }\end{array}$ & $\begin{array}{c}\text { LEEN } \\
\text { ORGANIOUE } \\
\text { ANEC } \\
\text { L'ESPACE }\end{array}$ \\
\hline FINALITE & $\begin{array}{l}\text { CAITEERES DE GULITÉ } \\
\text { EI } \\
\text { CATEEFES SCOLUX. }\end{array}$ & 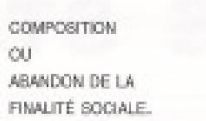 & 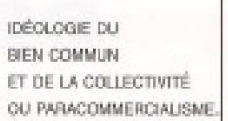 & $\begin{array}{l}\text { INTERET SOOAL } \\
\text { ET } \\
\text { INTERET LOCAL }\end{array}$ \\
\hline STRUCTURES & 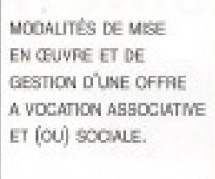 & 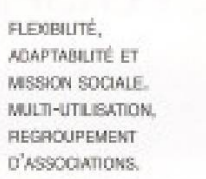 & $\begin{array}{l}\text { FINAHAEMENT DE LA } \\
\text { PIEFRE UENS WEC LES } \\
\text { IMSTIIUTIONS } \\
\text { A. WCCANON } \\
\text { SOCIALE. }\end{array}$ & $\begin{array}{l}\text { IMPLNTATION LOCLEF } \\
\text { OU TRLASFERE. } \\
\text { IMPACT DES POLTKUES } \\
\text { ALCIONALES ET } \\
\text { LOCLLES. }\end{array}$ \\
\hline ACTEURS & $\begin{array}{l}\text { ADHEAENTS, } \\
\text { HENEVOLES, SRLAFIES. } \\
\text { PHLOSOPHE DU HES } \\
\text { COMUUL. }\end{array}$ & 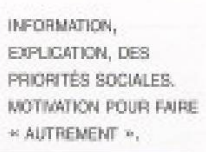 & 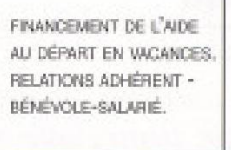 & 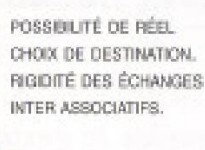 \\
\hline PROCÉDURES & 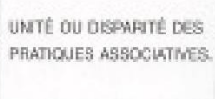 & 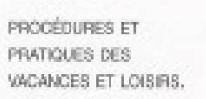 & $\begin{array}{l}\text { PEFTE OU FERFOFIENENT } \\
\text { DES PAMTILUES } \\
\text { ASSOCLTMLS. }\end{array}$ & PFoctouFES ET SITES. \\
\hline
\end{tabular}

20 Feld management : le terme Veld peut avoir deux traductions (USA) : rendement ; fléchissement, point limite. Le terme est apparu en hôtellerie dans l'article de Chevenak et Keane (1958) ; Vialle et Daudel, 1990.

\section{RÉFÉRENCES}

Balfet, Christiane, et al. (1994), Marketing dans les industries de l'accuell, Paris, B.P.I.

Balfet, M. (1994), Recherche et champ specifique en Sciences de Gestion : qualité d'une recherche et enseignement professionnalisé, Mémoire d habilitation a la direction de recherche, Université de Toulouse 1 .

Balfet, M. (1996), L'audit marketing touristique, Paris, Economica.

Chaspoul, C. (1991), \& Il n"y a plus de saisons of, Cahiers Espaces, n 109.

Chazaud, P. (1991), « Saisonnalité et aménagement du temps *, Cahiers Espaces, $\mathrm{n}^{\circ} 109$.

Chevenak et Keane (1958), « Yield management What, how and why w, DKC Report. The Hotel Technology news letter. Comell University, Ithaca, New York, avril.

Evrard, Y., et al, (1994), Market ; Erudes et recherches en Marketing, Paris, Nathan.

Froidure, Jean (1997), Du tourisme social au tourisme associatif, crises er mutations des as sociations françaises de tourisme, Paris, LHarmattan.
La Documentation Française (1990), Mémento du tourisme.

Lafforgue, A. (1994), * Commentaire du décret d'application de la loi du 13 juillet $1992 \%, \mathrm{Ca}=$ hiers Espaces, n'129.

Lassegue, J. (1981), Qu'est-ce que la Gestion? Mélange à Pierre Vigreux, Toulouse, I.A.E.

Lemoigne, J. L. (1993), * Sur l'incongruité épistemologique des Sciences de Gestion $\%$, Revue Française de Gestion, $\mathrm{n}^{\circ} 96$.

Marchesnay, M. (1991), De la theorisation en Sciences de Gestion ERFI, Montpellier, E.R.F.I.

Martinet, A.C., et all (1990), Epistémologie et Sciences de Géstion, Paris, Economica.

Porter, M. (1993), L'avantage concurrentiel des nations, Paris, Inter Editions.

Riveline, C. (1991), a De l'urgence en Ges-

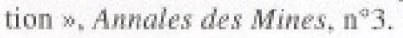

Tinand, Y. (1994), Le tourisme, economie et management, Paris, Edisciences.

Tocquer, G., et Zins, M. (1987), Marketing du Tourisme, Montréal, Gaétan Morin Editeur.

Treboul, J. B. (1998), Les strategies des entreprises de tourisme, Paris, PUF (Que sais-je ?).

Vellas, F, (1992), Le tourisme, Paris, Economica,

Vialle, G, et Daudel, S. (1990), Le Yeld managenew. La face encore cache du marketing des services, Paris, InterEditions. 